Case Report

\title{
Mycobacterium avium Complex-Associated Hemophagocytic Lymphohistiocytosis in a Sickle Cell Patient: An Unusual Fatal Association
}

\author{
Mohammed A. R. Chamsi-Pasha, ${ }^{1}$ M. Chadi Alraies, ${ }^{2}$ \\ Abdul Hamid Alraiyes, ${ }^{3}$ and Eric D. Hsi ${ }^{4}$ \\ ${ }^{1}$ Department of Internal Medicine, Cleveland Clinic, 9500 Euclid Avenue, Cleveland, OH 44195, USA \\ ${ }^{2}$ Cleveland Clinic Lerner College of Medicine of Case Western Reserve University, Department of Hospital Medicine, \\ Cleveland Clinic, 9500 Euclid Avenue, Cleveland, OH 44195, USA \\ ${ }^{3}$ Department of Pulmonary, Critical Care and Environmental Medicine, Tulane University Health Sciences Center, \\ 1430 Tulane Avenue, SL-9, New Orleans, LA 70112, USA \\ ${ }^{4}$ Section Head, Hematopathology, Chairman, Department of Clinical Pathology, Cleveland Clinic, \\ 9500 Euclid Avenue Cleveland, OH 44195, USA \\ Correspondence should be addressed to Mohammed A. R. Chamsi-Pasha; drpasha.moh@gmail.com
}

Received 13 March 2013; Accepted 28 April 2013

Academic Editors: D. Galanakis, K. Konstantopoulos, and Y. Matsukawa

Copyright (C) 2013 Mohammed A. R. Chamsi-Pasha et al. This is an open access article distributed under the Creative Commons Attribution License, which permits unrestricted use, distribution, and reproduction in any medium, provided the original work is properly cited.

Hemophagocytic lymphohistiocytosis (HLH) is a rare hyperinflammatory syndrome, characterized clinically by fever, splenomegaly, cytopenia, and high ferritin. Infectious causes have been associated with secondary HLH, with viruses being the most common. We report a case of Mycobacterium avium complex-associated HLH in a sickle cell anemia patient. To the best of our knowledge, this association has never been reported in sickle cell anemia.

\section{Introduction}

HLH is a rare inflammatory disease caused by macrophage and cytotoxic T-cell activation leading to uncontrolled hemophagocytosis and cytokine production. It can be divided into primary (genetic) causes, idiopathic, and secondary causes associated with infections, hematological malignancies, and autoimmune diseases [1]. Mycobacterium avium complex (MAC) is a nontuberculous mycobacterium known to cause pulmonary and disseminated disease in patients with immunodeficiency. We report the first case of $\mathrm{HLH}$ in the setting of MAC infection in a sickle cell anemia patient, initially presenting with fever of unknown origin.

\section{Case Presentation}

A 22-year-old female with sickle cell anemia presented with 1-month history of fever, night sweats, and weight loss. On presentation, vital signs were stable except for fever of $102 \mathrm{~F}$.
Cardiopulmonary examination was normal, and there was no lymphadenopathy. The rest of physical examination was unremarkable. Laboratory studies showed leukocytosis of 17.000/uL and hemoglobin of $10.4 \mathrm{~g} / \mathrm{dL}$. Erythrocyte sedimentation rate and C-reactive protein were elevated at $44 \mathrm{~mm} / \mathrm{hr}$ and $8.47 \mathrm{mg} / \mathrm{dL}$, respectively. Patient had negative bacterial and fungal cultures, viral serologies, and tuberculin skin testing. Chest X-ray showed bilateral nodular opacities, and computed tomography $(\mathrm{CT})$ revealed ground glass opacity in the left lower lobe with surrounding nodules (Figure 1). Bronchoalveolar lavage with biopsies showed granulomas with no organisms. Wedge biopsy was pursued and histopathology showed necrotizing granulomas (Figure 2(a)) with acid-fast bacilli (Figure 2(b)). Polymerase chain reaction and tissue cultures were positive for MAC, and patient was started on azithromycin, ethambutol, and rifampin.

One month after discharge, she was readmitted with high fevers and hypoxia concerning for severe sepsis. Labs showed leukocytosis of $35.000 / \mathrm{uL}$, alanine transaminase 


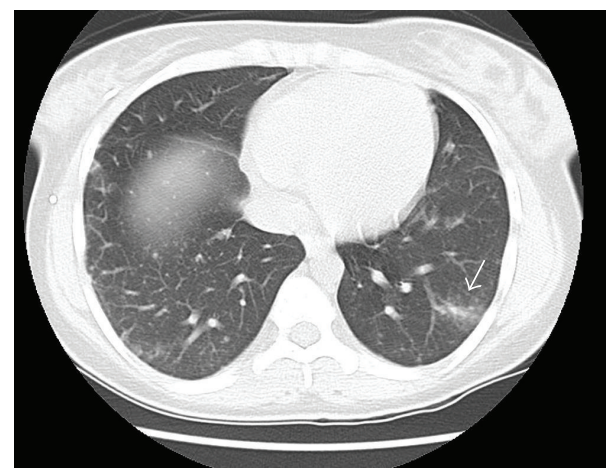

Figure 1: Compute tomography (CT) of chest showing wedgeshaped ground glass opacity in the left lower lobe, with surrounding centrilobular nodules (arrow). Additional 1-2 mm centrilobular nodules noted in the right lower lobe.

$224 \mathrm{U} / \mathrm{L}$, aspartate transaminase $592 \mathrm{U} / \mathrm{L}$, alkaline phosphatase $480 \mathrm{U} / \mathrm{L}$, and cholestatic jaundice (total bilirubin $10.2 \mathrm{mg} / \mathrm{dL}$ ). Severe sepsis was suspected, and patient was placed empirically on moxifloxacin, amikacin, azithromycin, and rifampin. CT of the chest showed multifocal pneumonia. Infectious workup revealed MAC in the blood with negative viral and bacterial cultures. Her course was complicated by persistent fevers, splenomegaly, and development of disseminated intravascular coagulopathy (DIC). An elevated ferritin of $38,539 \mathrm{ng} / \mathrm{mL}$ and triglyceride of $566 \mathrm{mg} / \mathrm{dL}$ were noted. Bone marrow biopsy showed histiocytes with engulfed erythrocytes and nucleated marrow elements (Figure 2(c)), along with scattered granulomas (Figure 2(d)). Bone marrow cultures were positive for MAC. A diagnosis of MACassociated HLH was made, and patient was started on methylprednisolone and interleukin-1 receptor antagonist (Anakinra) with immediate cessation of fever spikes. However, the patient's respiratory status deteriorated and passed away soon after.

\section{Discussion}

$\mathrm{HLH}$ is a rare syndrome resulting in massive activation of macrophages and cytotoxic T cells. Diagnosis is established if five out of eight criteria are met: fever; splenomegaly; cytopenia involving at least two lines; increased triglycerides and/or decreased fibrinogen; hemophagocytosis in a bone marrow, liver, or node specimen; low NK-cell activity; ferritin more than $500 \mathrm{ng} / \mathrm{mL}$; and elevated soluble interleukin 2 receptor [2]. Our patient's ferritin value of $38,539 \mathrm{ng} / \mathrm{mL}$ strongly supports the diagnosis of HLH, and the presence of hemophagocytic cells on bone marrow biopsy is consistent with this condition.

HLH has been associated with infections, with EpsteinBarr virus being the most common [1]. Rare cases of nontuberculous mycobacterial infections have been reported in association with HLH [3], but to date we present the first case of MAC-associated HLH in sickle cell patient.

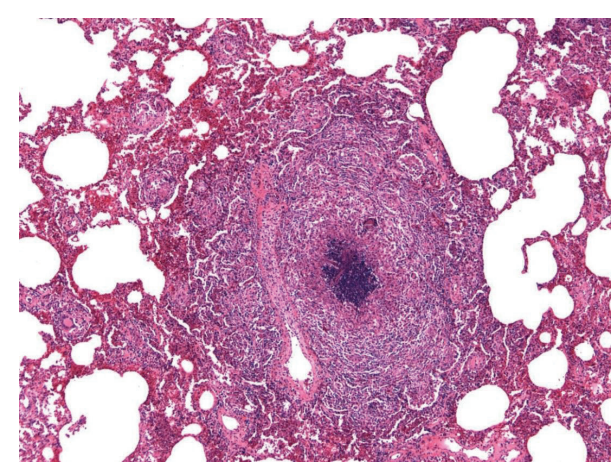

(a)

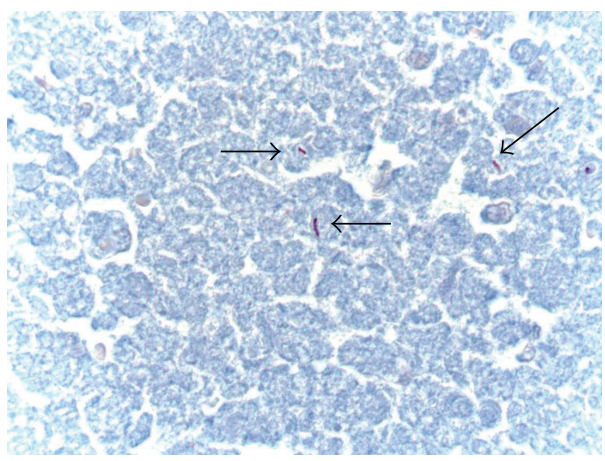

(b)

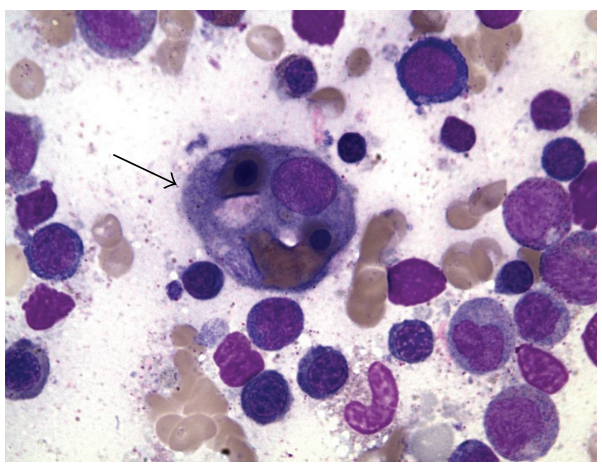

(c)

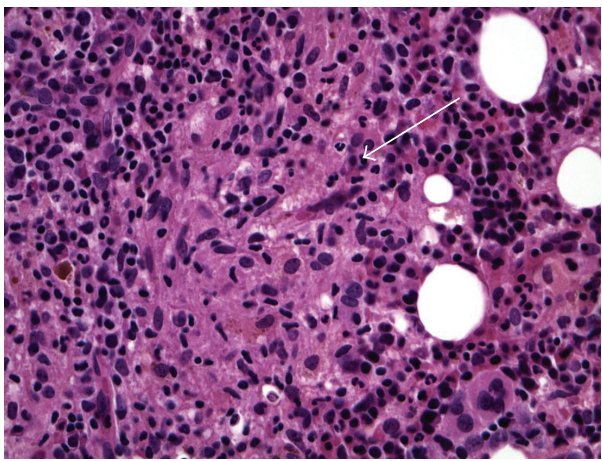

(d)

Figure 2: (a) Necrotizing granuloma evident on wedge-resected lung tissue. (b) Ziehl-Neelsen stain revealing acid-fast bacilli in resected lung tissue, consistent with mycobacteria (arrows). (c) Bone marrow aspirate specimen showing a hemophagocyte containing erythrocytes and pronormoblasts (arrow) (Wright stain, 1000x). (d) Core bone marrow showing a loose Granuloma (arrow) (Hematoxylin-Eosin stain, 400x). 
Disseminated MAC is a common opportunistic infection in HIV-infected or immunodeficient patients but never reported in adult sickle cell anemia patients.

There is no gold standard therapeutic regimen for secondary HLH. The aim is to suppress the inflammatory response using immunosuppressive agents. However, treating the underlying disease is necessary to expedite recovery and response to therapy [1]. Agents adopted by the Histiocyte Society include combination of dexamethasone, etoposide, and intrathecal methotrexate [2]. In our case, high bilirubin levels and DIC precluded the use of etoposide and intrathecal methotrexate, respectively. The patient was started on interleukin-1 receptor antagonist, which has been shown to improve symptoms in autoinflammatory diseases [2].

This case aims to increase clinician's awareness of HLH in the setting of infectious process, as mortality rates are high and early diagnosis and treatment are mandated to improve outcomes [4].

\section{Conflict of Interests}

The authors have no conflict of interests to declare.

\section{References}

[1] N. G. Rouphael, N. J. Talati, C. Vaughan, K. Cunningham, R. Moreira, and C. Gould, "Infections associated with haemophagocytic syndrome," Lancet Infectious Diseases, vol. 7, no. 12, pp. 814-822, 2007.

[2] C. Larroche, "Hemophagocytic lymphohistiocytosis in adults: diagnosis and treatment," Joint Bone Spine, vol. 79, pp. 356-361, 2012.

[3] Y. H. Chou, M. S. Hsu, W. H. Sheng, and S. C. Chang, "Disseminated Mycobacterium kansasii infection associated with hemophagocytic syndrome," International Journal of Infectious Diseases, vol. 14, no. 3, pp. e262-e264, 2010.

[4] A. Hot and J. Ninet, "Healing hemophagocytic syndrome in adults: the challenge continues," Clinical Advances in Hematology and Oncology, vol. 6, no. 8, pp. 591-593, 2008. 


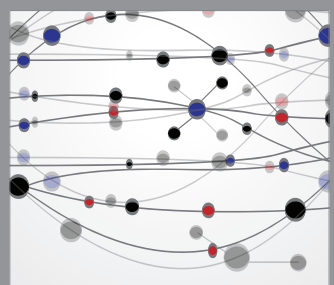

The Scientific World Journal
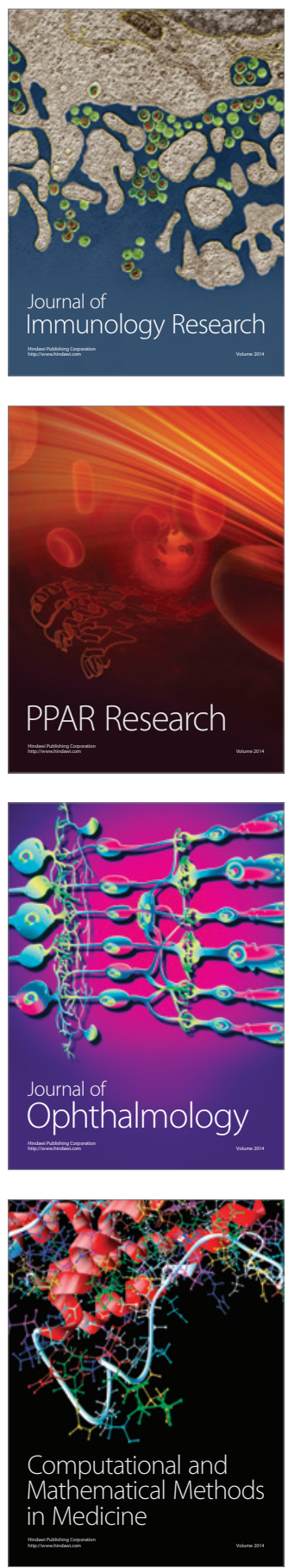

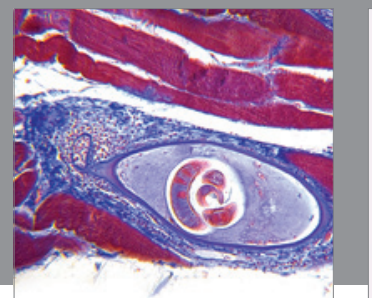

Gastroenterology

Research and Practice
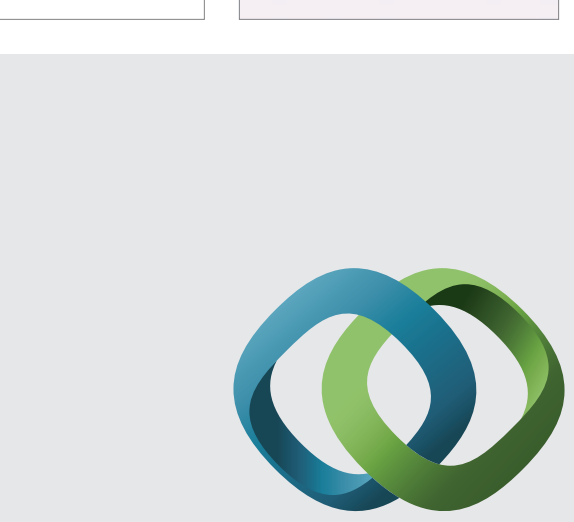

\section{Hindawi}

Submit your manuscripts at

http://www.hindawi.com
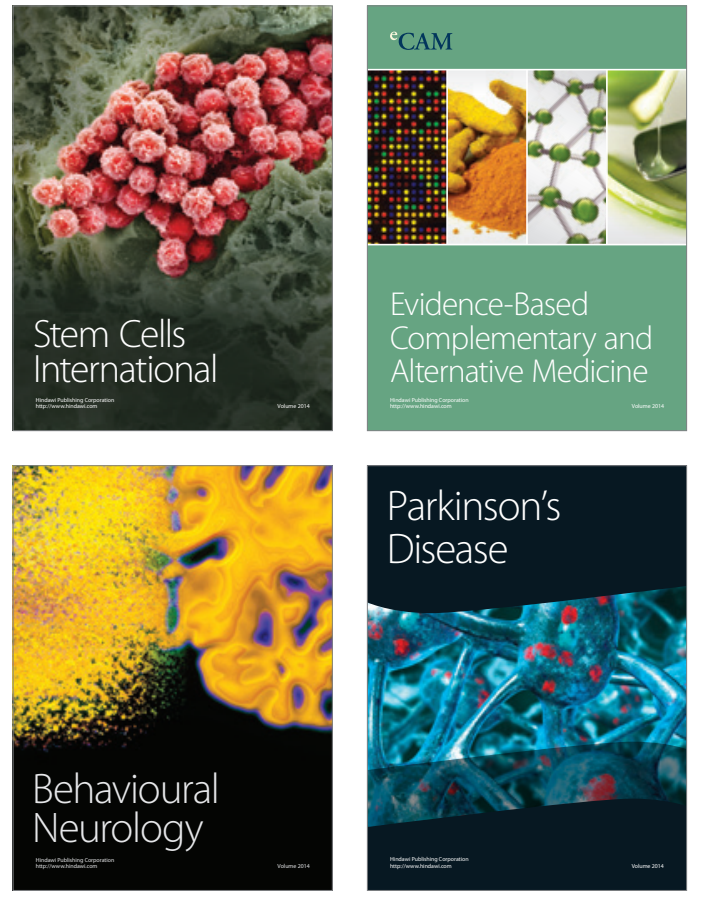
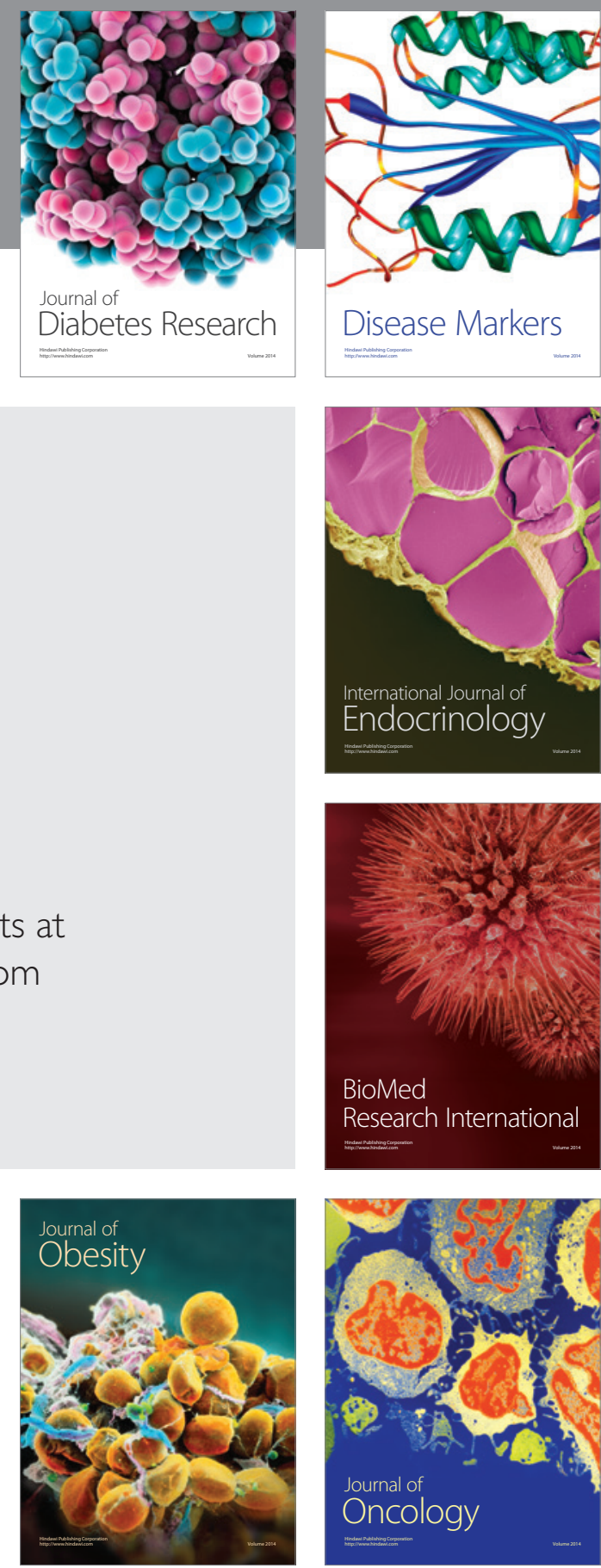

Disease Markers
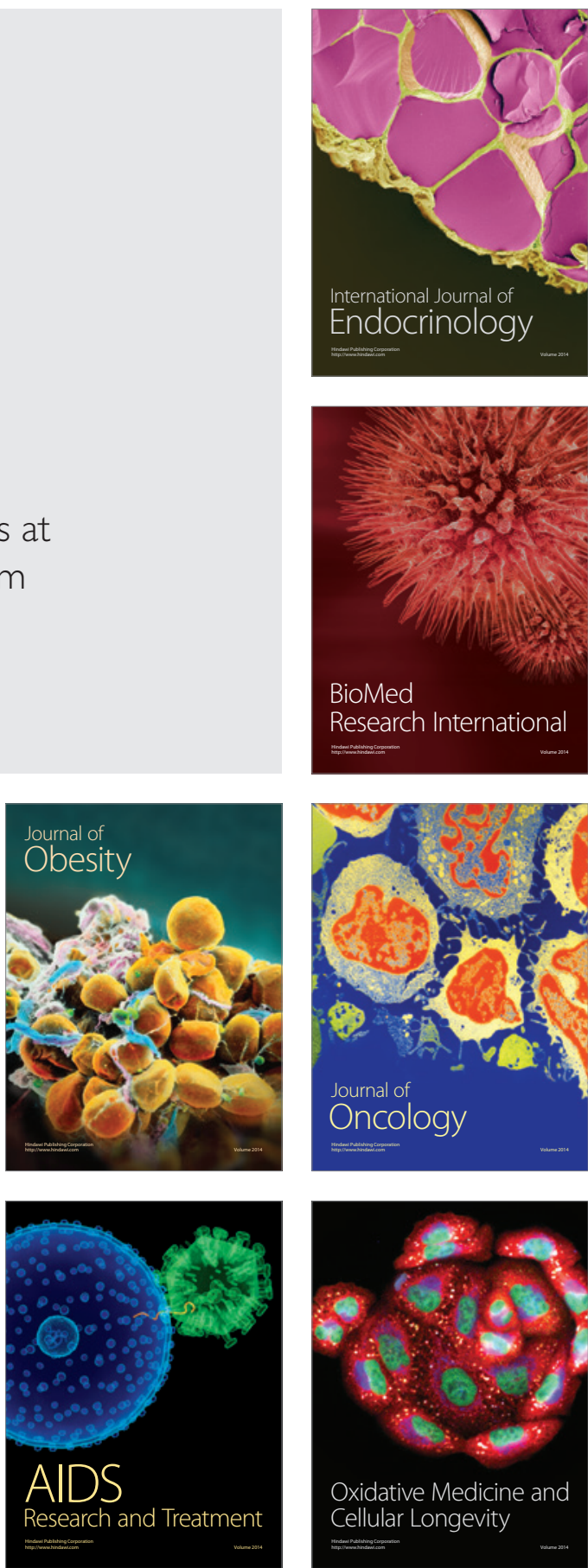